\title{
OPERATOR MINIMAX THEOREMS IN BANACH LATTICES
}

\author{
CARL G. LOONEY
}

\begin{abstract}
Let $\psi: X \times Y \rightarrow(E,<,\|\cdot\|)$, where $X$ and $Y$ are convex, $X$ is compact, and $E$ is a dedekind complete Banach lattice with unit $e$. If each $\psi(\cdot, y)$ is continuous <-concave on $X,\{\psi(\cdot, y): y \in Y\}$ is convex, and $\psi(X \times Y)$ is minorized in $E$, then sup inf $\psi(x, y)=\inf \sup \psi(x, y)$. Similar theorems are included.
\end{abstract}

1. Introduction. An ordered vector space is a vector space $V$ with a partial ordering $\leqslant$ defined on it such that $f \leqslant g$ in $V$ implies that (i) $f+h \leqslant g+h$ for all $h \in V$, and (ii) $\alpha f \leqslant \alpha g$ for all $\alpha \geqslant 0$. If $V$ satisfies (iii) $\sup (f, g)$ and $\inf (f, g)$ are defined and belong to $V$ for any $f, g$ in $V$, then $V$ is called a vector lattice (or Riesz space). A vector lattice $V$ is dedekind complete [7] (or boundedly order complete) iff every subset $S$ of $V$ which is majorized (i.e., there is some $v$ in $V$ such that $s \leqslant v$ for every $s$ in $S$, also called an upper bound for $S)$ has a supremum $\sup (S)$ in $v$.

For any $v \in V, v^{+}=\sup (v, 0)$ and $v^{-}=-\inf (v, 0)$ are known as the positive and negative parts of $v$, respectively, while $V^{+}=\left\{v^{+}: v \in V\right\}$ is the positive cone of $V$. We note that $V=V^{+}-V^{+}$when $V$ is a vector lattice. The absolute value of $v$ is defined to be $|v|=v^{+}+v^{-}$.

$(E, \leqslant,\|\cdot\|)$ is a Banach lattice iff $(E, \leqslant)$ is a vector lattice, $(E,\|\cdot\|)$ is a Banach space, and the norm and absolute value are related via: $|f|>|g|$ implies that $\|f\| \geqslant\|g\|[6$, p. 236]. For our purposes we note that the set $C_{E}(T)$ of all continuous functions on a compact set $T$ into a Banach lattice $E$ is a Banach lattice (see [10] for further examples). If $C_{E}(T)$ is dedekind complete and completely regular, then $T$ is extremally disconnected (see [10, p. 15] or [1, vol. III, p. 82]).

Now let $E$ be a Banach lattice with positive cone $E^{+}$and let $E^{\prime}$ be the space of all algebraic linear functionals on $E$. If $\left(E^{\prime}\right)^{+}$is the positive cone in $E^{\prime}$, then $E^{\circledast} \equiv\left(E^{\prime}\right)^{+}-\left(E^{\prime}\right)^{+}$is the order dual of $E$. It is well known that any $L \in\left(E^{\prime}\right)^{+}$is continuous ( $L$ is bounded on the order bounded set $U^{+}=U$ $\cap E^{+}$, where $U$ is the unit ball, and so on $U=U^{+}-U^{+}$), and thus $E^{\circledast} \subset E^{*} \equiv$ space of continuous linear functionals on $E$ (see $[10$, p. 86] for a more general result). We say that $e \in E$ is an order unit iff for each $x \in E$ there is an $\alpha>0$ such that $\alpha e \geqslant x$, e.g., the constant function 1 in $C[0,1]$.

Received by the editors August 6, 1976.

AMS (MOS) subject classifications (1970). Primary 49A40; Secondary 47B55, 46A05.

Key words and phrases. Minimax theorem, Banach lattice, convex operator, convex sets, quasiconvex operators, Dinculeanu representation theorem.

(c) American Mathematical Socicty 1977 
2. Let $\psi: A \rightarrow(E, \leqslant)$, where $A$ is a convex set and $(E, \leqslant)$ is a vector lattice. We will call $\psi \mathrm{a} \leqslant$-convex operator iff $\psi(\alpha f+(1-\alpha) g) \leqslant \alpha \psi(f)+$ $(1-\alpha) \psi(g)$ for any $f, g \in A$ and $0 \leqslant \alpha \leqslant 1$ and will say that $\psi$ is $\leqslant-$ concave whenever $-\psi$ is $\leqslant$-convex. This is more general than the linear case of strict equality (we will call a $\leqslant$-convex function order convex).

TheOREM 1. Let $\psi: X \times Y \rightarrow(E, \leqslant,\|\cdot\|)$, where $X$ is a compact convex subset of a normed space, $Y$ is a convex subset of a vector space, and $E$ is a dedekind complete Banach space whose positive cone $E^{+}$has nonempty interior. If (i) $x \rightarrow \psi(x, y)$ is continuous $\leqslant$-concave on $X$ for each fixed $y$, (ii) $y \rightarrow$ $\psi(x, y)$ is $\leqslant$-convex for each fixed $x$, and (iii) the image $\psi(X \times Y)$ is minorized in $E$, then

$$
\sup _{\boldsymbol{X}} \inf _{\boldsymbol{Y}} \psi(x, y)=\inf _{\boldsymbol{Y}} \sup _{\boldsymbol{X}} \psi(x, y) \text {. }
$$

Proof. For any fixed $\left(x_{0}, y_{0}\right), \inf _{Y} \psi\left(x_{0}, y\right) \leqslant \psi\left(x_{0} y_{0}\right) \leqslant \sup _{X} \psi\left(x, y_{0}\right)$. By taking the supremum over $X$ of both sides of $\inf _{Y} \psi\left(x_{0}, y\right) \leqslant \sup _{X} \psi\left(x, y_{0}\right)$, we obtain

$$
\sup _{X} \inf _{Y} \psi(x, y) \leqslant \sup _{X} \psi\left(x, y_{0}\right)
$$

Since this is true for any $y_{0}$ in $Y$, the inequality

$$
a \equiv \sup _{X} \inf _{Y} \psi(x, y) \leqslant \inf _{Y} \sup _{X} \psi(x, y) \equiv b
$$

always holds. Since each $\psi(\cdot, y)$ is continuous on $X$, it is majorized by $b_{y}=\sup _{X} \psi(x, y)$ in $E$ and hence $b=\inf \left\{b_{y}: y \in Y\right\}$ exists in $E$ by dedekind completeness. Since $\psi(X \times Y)$ is minorized in $E, a$ belongs to $E$. In the following paragraphs we establish that $a \geqslant b$, which will complete the proof.

The norm on $C_{E}(X)$ is the uniform norm $\|f\| \equiv \sup \left\{\|f(x)\|_{E}: x \in X\right\}$, where the norm on $E$ is designated by $\|\cdot\|_{E}$. We define $Q \equiv\left\{g \in C_{E}(X)\right.$ : $g \geqslant \psi(\cdot, y)$ on $X$ for some $y \in Y\}$.

Claim 1. $Q$ is convex. For any $g_{1}, g_{2} \in Q$ with $g_{i} \geqslant \psi\left(\cdot, y_{i}\right)(i=1,2)$, it follows by the convexity of $Y$ and the $\leqslant$-convexity of $y \rightarrow \psi(x, y)$ for each $x$ that

$$
\alpha g_{1}+(1-\alpha) g_{2} \geqslant \alpha \psi\left(\cdot, y_{1}\right)+(1-\alpha) \psi\left(\cdot, y_{2}\right) \geqslant \psi\left(\cdot, \alpha y_{1}+(1-\alpha) y_{2}\right) .
$$

Thus

$$
g_{3} \equiv \alpha g_{1}+(1-\alpha) g_{2} \geqslant \psi\left(\cdot, y_{3}\right)
$$

for $y_{3}=\alpha y_{1}+(1-\alpha) y_{2} \in Y$ and this implies that $g_{3} \in Q$.

Claim 2. $Q$ is a convex body. Since $E^{+}$has nonempty interior, we take $e$ to be an interior point of $E^{+}$. Denote by $\theta_{e}$ the constant function $\{x \rightarrow e\}$ in $C_{E}(X)$. Then $\theta_{e}$ is an interior point in $C_{E}(X)^{+}$(since for $\varepsilon>0$ sufficiently small that $N_{\varepsilon}(e) \equiv\left\{u \in E:\|e-u\|_{E}<\varepsilon\right\} \subset E^{+}$, the set $M_{\varepsilon}\left(\theta_{e}\right)=\{f \in$ $C_{E}(X): f(x) \in N_{\varepsilon}$ for all $\left.x \in X\right\}$ is a nbhd of $\theta_{e}$ and is contained in $C_{E}(X)^{+}$, because $g \in M_{\varepsilon}\left(\theta_{e}\right)$ implies that $g(x) \geqslant 0 \in E$ for every $\left.x \in X\right)$. Now we fix $y=y_{0}$ and put $h=\psi\left(\cdot, y_{0}\right)+e$ and then translate the cone $C_{E}(X)^{+}$to $h$ to 
get $P=C_{E}(X)^{+}+h$. Since $P \subset Q$ and $P$ has nonempty interior, $Q$ is a convex body.

Claim 3. The constant function $\{x \rightarrow b\}$, denoted by $\theta_{b}$, is not an interior point of $Q$. Because $\psi(X \times \mathrm{Y})$ is minorized, we may and do assume without loss of generality that $b>0$. Then $\theta_{b} \in C_{E}(X)^{+}$. Let $N_{\varepsilon} \equiv\left\{f \in C_{E}(X)\right.$ : $\left.\left\|f-\theta_{b}\right\|<\varepsilon\right\}$ and let $\theta_{u}=\{x \rightarrow u\}$ for $u \in E$ such that $\|b-u\|_{E}<\varepsilon$ (i.e., $\left\|\theta_{b}-\theta_{u}\right\|<\varepsilon$ ) and $b \geqslant u \geqslant 0$ with $b \neq u$. Now $b-u<b=$ $\inf _{Y} \sup _{X} \psi(x, y)$, so that $\theta_{b-u} \equiv\{x \rightarrow b-u\} \notin Q$.

Since $\theta_{b}$ is not an interior point of the convex body $Q$, the separation theorem yields the existence of a continuous linear functional $L \in C_{E}(X)^{*}$ which separates $Q$ and $\theta_{b}$. Thus $L\left(\theta_{b}\right) \leqslant \inf \{L(q) \in R: q \in Q\}$.

Claim 4. $L$ is positive. Let $g \in C_{E}(X)^{+}$and $d \in E^{+}$with $d=$ unit. Then for $\alpha$ sufficiently large, $L(\alpha(g+d)) \geqslant L(b)$, since $g$ is minorized on the compact set $X$. Whence $L(g+d) \geqslant(1 / \alpha) L(b) \geqslant \lim (1 / \alpha) L(b)=0$. Since $d$ is arbitrary in $E^{+}, L(g) \geqslant 0$.

$L$ is therefore a positive bounded function on $C_{E}(X)$. Hence $L$ is dominated (see Corollary 1 of $[2$, p. 387]). By the Dinculeanu representation theorem [2, p. 380] (or Corollary 2 of [2, p. 387]), there exists a regular Borel $E^{*}$-valued measure $m$ on the Borel sets in $X$ such that $L(f)=\int f d m$ for any $f \in C_{E}(X)$. Further $m$ has finite variation $\mu$, and we assume without loss of generality that $\mu(X)=1$. Now $b=b \mu(X)=\int \theta_{b} d \mu \leqslant \int g d \mu$ in $E$ for every $g \in Q$, by separation.

It is known that $\mu$ has a barycenter $\bar{x} \in X[8$, p. 2]. By Lemma 1 below and the $\leqslant$-concavity of each $\psi(\cdot, y)$, it follows that $\int \psi(\cdot, y) d \mu \leqslant \psi(\bar{x}, y)$ for each $y \in Y$. Then $b=\int \theta_{b} d \mu \leqslant \int \psi(\cdot, y) d \mu \leqslant \psi(\bar{x}, y)$ for each $y$ (since $\psi(\cdot, y) \in$ $Q$ in this continuous case), so that $b \leqslant \inf _{Y} \psi(\bar{x}, y) \leqslant \sup _{X} \inf _{Y} \psi(x, y)=a$. Thus $a=b$.

Lemma 1. Let $f: X \rightarrow E$ be u.s.c. $\leqslant$-concave on the compact convex set $X$ with $E$ a dedekind complete Banach space. If $\mu$ is a positive regular Borel measure on $X$ with $\mu(X)=1$ and $\bar{x} \equiv \int x d \mu \in X$ (the barycenter or $\mu$-mean of $X)$, then $f(\bar{x}) \geqslant \int f(x) d \mu$.

ProOF.

$$
\int x d \mu=\lim \sum_{1}^{n} x_{i} \mu\left(A_{i}\right)-\lim \sum_{1}^{n} \alpha_{i} x_{i},
$$

where $\mu\left(A_{i}\right) \equiv \alpha_{i}, \sum_{1}^{n} \alpha_{i}=1$, and the $A_{i}$ are disjoint Borel subsets which partition $X$. Then

$$
f(\bar{x})=f\left(\lim \sum \alpha_{i} x_{i}\right) \geqslant \lim \sup \left(\sum \alpha_{i} f\left(x_{i}\right)\right)=\int f(x) d \mu(x)
$$

by the $\leqslant$-concavity and the upper semicontinuity of $f$. This completes the proof of the lemma and the theorem.

Theorem 2. Let $\theta: X \times Y \rightarrow(E, \leqslant,\|\cdot\|)$, where $X$ and $Y$ are convex with $X$ being compact, and $E$ is a dedekind complete Banach lattice with interior 
( $\left.E^{+}\right)$nonempty. If (i) $X \rightarrow \theta(x, y)$ is continuous $\leqslant$-convex on $X$ for each fixed $y$, (ii) $y \rightarrow \theta(x, y)$ is $<$-concave for each fixed $x$, and (iii) $\theta(X \times Y)$ is majorized in $E$, then

$$
\sup _{Y} \inf _{X} \theta(x, y)=\inf _{X} \sup _{Y} \theta(x, y) .
$$

3. Let $F: X \rightarrow(E, \leqslant,\|\cdot\|)$ where $X$ is convex and $E$ is a dedekind complete Banach lattice. We will call $f \leqslant-$ quasiconvex iff $f(\alpha x+(1-\alpha) y)$ $<\sup (f(x), f(y))$ for any $x, y \in X$ and $0 \leqslant \alpha \leqslant 1$. In this case $-f$ is $<$-quasiconcave.

LeMma 2. The mapping $f: X \rightarrow(E, \leqslant,\|\cdot\|)$ is $\leqslant$-quasiconvex on $X$ iff every set of the form $K_{d} \equiv\{x \in X: f(x) \leqslant d \in E\}$ is convex.

Proof. Let $f$ be $\leqslant$-quasiconvex. For any $d \in E$, let $x_{1}$ and $x_{2}$ belong to $K_{d}$ and $0<\alpha<1$. Then $f\left(\alpha x_{1}+(1-\alpha) x_{2}\right) \leqslant \sup \left(f\left(x_{1}\right), f\left(x_{2}\right)\right) \leqslant d$, which implies that $\alpha x_{1}+(1-\alpha) x_{2} \in K_{d}$. Thus $K_{d}$ is convex.

Now assume that $K_{d}$ is convex for any $d \in E$ and let $x, y \in X$ with $0<\alpha<1$. Let $c=\sup (f(x), f(y))$. Then $x, y, \alpha x+(1-\alpha) y \in K_{c}$, from which it follows that $f(\alpha x+(1-\alpha) y) \leqslant c=\sup (f(x), f(y))$.

Lemma 3. If $f: X \rightarrow(E, \leqslant,\|\cdot\|)$ is $\leqslant$-convex, then $f$ is $\leqslant$-quasiconvex.

Proof. Since

$$
\begin{aligned}
f(\alpha x+(1-\alpha) y) & \leqslant \alpha f(x)+(1-\alpha) f(y) \\
\leqslant & \alpha \sup (f(x), f(y))+(1-\alpha) \sup (f(x), f(y)) \\
= & \sup (f(x), f(y)),
\end{aligned}
$$

$\leqslant$-quasiconvexity is more general than $\leqslant$-convexity.

We conjecture here that if condition (ii) in Theorem 1 were changed from $\leqslant$-convexity to $\leqslant$-quasiconvexity, then the theorem would remain true.

TheOREM 3. Let $\psi: X \times Y \rightarrow(E, \leqslant,\|\cdot\|)$, where $X$ is compact convex, $Y \equiv$ any set, and $E$ is a dedekind complete Banach lattice with order unit $e$. If (i) $x \rightarrow \psi(x, y)$ is continuous $\leqslant$-concave on $X$ for each $y$, (ii) $G \equiv\{\psi(\cdot, y)$ : $y \in Y\}$ is convex, and (iii) $\psi(X \times Y)$ is minorized in $E$, then

$$
\sup _{X} \inf _{Y} \psi(x, y)=\inf _{Y} \sup _{X} \psi(x, y) \text {. }
$$

Proof. It suffices to prove Claim 1 in the proof of Theorem 1 under the new hypothesis (since $e \in E^{+}$is sufficient to establish Claim 2). But

$$
g_{3} \equiv \alpha g_{1}+(1-\alpha) g_{2} \geqslant \alpha \psi\left(\cdot, y_{1}\right)+(1-\alpha) \psi\left(\cdot, y_{2}\right) \equiv \psi\left(\cdot, y_{3}\right) \in G
$$

does this, and thus proves the theorem.

THEOREM 4. Let the hypotheses of Theorem 3 be changed thusly: (i) $x \rightarrow$ $\psi(x, y)$ is continuous $\leqslant$-convex for each $y$ and (iii) $\psi(X \times Y)$ is majorized in $E$. Then

$$
\inf _{X} \sup _{Y} \psi(x, y)=\sup _{Y} \inf _{X} \psi(x, y) \text {. }
$$


4. An example of an order convex operator is: $Q: C[0,1]^{+} \rightarrow C[0,1]^{+}$via $[Q(f)](t)=\int_{0}^{t} f^{2}(s) d s$. For $0<f, g$,

$$
\int_{0}^{t}[\alpha f(s)+(1-\alpha) g(s)]^{2} d s \leqslant \alpha \int_{0}^{t} f(s)^{2} d s+(1-\alpha) \int_{0}^{t} g(s)^{2} d s
$$

by the convexity of $t \rightarrow t^{2}$ and the positivity of the integrands.

An example of a Banach lattice whose positive cone has empty interior is the space $l_{1}$ of all absolutely summable sequences of real numbers. Let $\left(x_{1}, x_{2}, \ldots\right) \in l_{1}^{+}$and $N_{\varepsilon}=\left\{\left(y_{1}, y_{2}, \ldots\right): \sum_{i=1}^{\infty}\left|x_{i}-y_{i}\right|<\varepsilon\right\}$. Then take $z=\left(z_{1}, z_{2}, \ldots\right)$, where $z_{i}=x_{i}$ for all $i$ except $i=N$ with $x_{N}<\varepsilon / 3$, and put $z_{N}=-x_{N}$. But

$$
\|z-x\|_{1}=\sum_{i=1}^{\infty}\left|z_{i}-x_{i}\right|=\left|z_{N}-x_{N}\right|<2 \varepsilon / 3
$$

Thus $z \in N_{\varepsilon}$ but $z \notin l_{1}^{+}$.

While $l_{1}$ is a dedekind complete Banach lattice, it has no order unit. Put $x^{(k)}=(0,0, \ldots, 1,0, \ldots)$ with 1 in the $k$ th position. Then $\left(x^{(k)}\right)$ is a sequence in the closed unit ball $B_{1}(0)$, but $\sup \left\{x^{(k)}: k=1,2, \ldots\right\}=(1,1$, $1 \ldots) \notin l_{1}$. While $\left(x^{k}\right)$ is bounded, it is not order bounded. If $e$ were an order element in $l_{1}$, then for $\alpha=1 /\|e\|_{1}$, $\alpha e$ would have norm 1 and be a unit element and thus $x^{(k)} \leqslant \alpha e$ for all $k$, so that $\sup \left\{x^{(k)}\right\} \leqslant \alpha e$, a contradiction.

However, $l_{\infty}$ is a dedekind complete Banach lattice with unit element $e=(1,1, \ldots) . C[0,1] \equiv$ space of continuous real valued functions on $[0,1]$ is a Banach lattice with unit $e(x)=1(0 \leqslant x \leqslant 1)$ but it is not dedekind complete $\left(\|\cdot\|=\right.$ supremum norm). Its order completion, $L^{\infty}[0,1]$, is dedekind complete.

The class of Banach lattices with positive cones having nonempty interior (any interior point is an order unit) which are dedekind complete is limited. Thus we would hope to have weaker conditions on Theorem 1. The next formulation has weaker, but more vague, conditions.

TheOREM 5. Let $\psi: X \times Y \rightarrow(E, \leqslant,\|\cdot\|)$, where $X$ is a compact convex subset of a normed space, $Y$ is any set, and $E$ is a Banach lattice with unit element 1. If

(i) $x \rightarrow \psi(x, y)$ is continuous order concave,

(ii) $G=\{\psi(\cdot, y): y \in Y\}$ is convex, and

(iii) $\psi(X \times Y)$ is minorized in $E$, then

$$
a \equiv \sup _{X} \inf _{Y} \psi(x, y)=\inf _{Y} \sup _{X} \psi(x, y) \equiv b
$$

whenever $a$ and $b$ exist in $E$.

An analogous result holds as before.

\section{REFERENCES}

1. G. Choquet, Lectures on analysis. Vols. I, II, III, Benjamin, New York, 1969. 
2. N. Dinculeanu, Vector measures, Pergamon, Berlin, 1967.

3. Ky Fan, Minimax theorems, Proc. Nat. Acad. Sci. U.S.A. 39 (1953), 42-47.

4. G. Jameson, Ordered linear spaces, Lecture Notes in Math., vol. 141, Springer-Verlag, Berlin, 1970.

5. H. Kneser, Sur un théorème fondamental de la théorie de jeux, C. R. Acad. Sci. Paris 234 (1952), 2418-2420.

6. J. L. Kelley, I. Namioka et al., Linear topological spaces, Van Nostrand, Princeton, N.J., 1963.

7. W. A. J. Luxemburg and A. C. Zaanen, Riesz spaces. Vol. I, North-Holland, Amsterdam, 1971.

8. R. R. Phelps, Lectures on Choquet's theorem, Van Nostrand Math. Studies No. 7, Van Nostrand, New. York, 1966.

9. J. von Neuman, Zur theorie der Gellschaftsspiele, Math. Ann. 100 (1928), 295-320.

10. A. L. Peressini, Ordered topological vector spaces, Harper and Row, New York, 1967.

DePartment of Mathematics, University of TOledo, Toledo, OHIO 43606 